Vasquez, M, Haworth, B, Hopkinson, N (2011) Optimum sintering region for laser sintered nylon-12, PROCEEDINGS OF THE INSTITUTION OF MECHANICAL ENGINEERS PART B-JOURNAL OF ENGINEERING MANUFACTURE, 225(B12), pp.2240-2248, ISSN: 0954-4054. DOI: 10.1177/0954405411414994.

\title{
Optimum sintering region for laser sintered Nylon-12
}

\author{
Abstract \\ Previous work has revealed that mechanical properties in laser sintered Nylon-12 parts \\ improve with increased applied energy, which promotes more complete melting and \\ consolidation in the parts (Hopkinson 2009). However, Caulfield (2006) showed that this \\ trend reaches a maximum after which the addition of more energy into the part causes \\ mechanical properties to decline. It is thought that the reason for this decline is that the \\ additional energy causes polymer chain degradation or other changes in molecular \\ structure. This paper aims to use thermogravimetric analysis (TGA) to investigate the \\ thermal degradation of Nylon-12 and explain the deterioration of mechanical properties \\ when high energy density conditions are applied during processing. The key findings are \\ the application of modelling methods to predict the temperatures achieved during laser \\ sintering of Nylon-12. It is shown that temperatures in the laser sintering machine can \\ achieve levels above $300^{\circ} \mathrm{C}$. At these temperatures TGA data shows that mass loss occurs
}


and could cause mechanical property breakdown. This practical work coupled differential scanning calorimetry (DSC) and thermogravimetric analysis as a means of identifying thermal transitions in the material. The term 'Stable Sintering Region' is proposed as a novel concept for the laser sintering community and can have implications for better understanding of how process parameters can affect parts built in the machine. In addition, the concept could be used in the material selection process when screening potential new polymers for the process. One limitation of laser sintering, compared to other polymer processes such as injection moulding, is the limited of understanding of the connection between machine parameters and part properties. This work aims to improve that understanding by discussing the pattern of thermal behaviour, including degradation, seen in polyamide exposed to high laser parameters.

Keywords Additive Manufacturing, Thermal Degradation, TGA, Stable Sintering Region, Laser Sintering Window

\section{Introduction}

Laser sintering is an additive manufacturing technology that utilizes powdered metal, ceramic, or polymer materials to construct prototype and end use 3-D parts. Compared to other additive manufacturing technologies laser sintering can employ a wide range of 
materials. The focus of this paper will be on polymer laser sintering, for which the available range of materials is relatively limited, in comparison to competitive processes such as injection moulding. Nylon-12 is the most common material used in polymer laser sintering. The material has been used in a multitude of applications including in the aerospace industry, for ducting in fighter aircraft, and in the sports industry for rideable snowboard binding prototypes (Hopkinson 2005).

Laser sintering has several processing variables that can be independently set and controlled by the operator to deliver parts with strong and repeatable properties. The main settings that are responsible for part property control are laser power, laser scan count, scan spacing, and part bed temperature. In a normal nylon-12 laser sintering build the part bed is heated to a temperature range just below the melting temperature range of the polymer. This helps to limit part distortion and aids sintering initiation once the laser interacts with the powder (Zarringhalam 2009). A minimum amount of energy is needed to create parts however; it has been shown that there is a range above this lower input energy that still enables the construction of viable parts. The more energy that is introduced into the system either by laser power, bed temperature, or scan count can create a more dense part. One of the downsides of adding too much energy to the sintering region is that there will be spill- 
over into areas near the part. This can cause support material to be caked to the part, making part breakout difficult.

Caulfield (2007) has shown that increasing the laser sintering energy density (Equation 1) increases part properties in Nylon-12 (Figure 1). This effect reaches a maximum after which properties decline. This phenomenon has also been seen in laser sintering of polycarbonate (Ho 1999). The authors do not focus on this drop off in properties other than suggesting that the high energy density causes a deterioration of the polymer chain. Another reason for the decline of properties is that the excess energy being absorbed by the polymer is enough to locally vaporize the material, causing gas bubbles to form which can lead to part porosity. The greater the volume of gas, the higher the potential for voids. In addition, if the molecular structure of the polymer is changed at high density input, this will influence physical properties (viscosity; surface energy) that are known to underpin the sintering behaviour, in this process.

$$
U_{E}=\frac{P}{V_{S} \times V_{B}}
$$

where 
$\mathrm{U}_{\mathrm{E}}=$ Energy density $\left(\mathrm{J} \mathrm{mm}^{-2}\right), \mathrm{P}=$ laser power $(\mathrm{W}), \mathrm{y}_{\mathrm{S}}=$ scan spacing $(\mathrm{m}) \mathrm{v}_{\mathrm{B}}=$ beam speed $(\mathrm{m}$ $\left.\mathrm{s}^{-1}\right)$

A precursor to this work was completed by Williams and Deckard (1998) investigating advances in modelling the effect of selected parameters on the SLS process. The researchers used process-defined parameters to estimate their effect on mechanical properties such as density and modulus and outlined a methodology for modelling temperatures achieved in the process for Bisphenol-A polycarbonate (PC). The paper mentions that degradation can occur in the process and incorporates the specific kinetics for polycarbonate in terms of heat loss resulting from the endothermic process of depolymerisation (Williams 1998). However, there was no attempt to define an exact region of optimal sintering for this material using degradation measurement techniques such as TGA.

For most polymers there is no discrete point of degradation but previous work relating to other polymer processes such as injection moulding has established thermal processing regions, defined partly on this basis. Colin (2006) defined a processability window using a combination of temperature and molar mass graphs. The main factors for these plots are the 
ductile-brittle critical molar mass, the thermal stability ceiling, and a minimum 'fluidity temperature' to control viscosity. Thermal stability is not easily defined, since the onset of degradation depends upon the chemical mechanism involved (hydrolysis; thermooxidation) and factors other than temperature, including process residence time and mechanical stress.

Using a combination of thermogravimetric and gas chromatographic techniques, together with kinetic analysis software, there have been attempts by Herrara and Ghosh (2006) to outline the chemical reactions that occur during degradation for polymers such as polyamide. The exact kinetics of degradation for laser sintering are not discussed here.

The goal of this paper is to quantify a temperature range for optimal sintering of nylon-12 to avoid polymer degradation and reduction of part properties. This work will aim to connect the observations made by Caulfield with in-process laser sintering temperature calculations and experimental thermogravimetric analysis of nylon-12 powder in atmospheric conditions simulating the laser sintering process. In particular, the work reported will be used to propose a new concept, in terms of a 'stable sintering region' and hence a 'laser sintering window', for semi-crystalline polymers such as nylon-12, to help to 
enhance the understanding of relationships between material properties and process conditions.

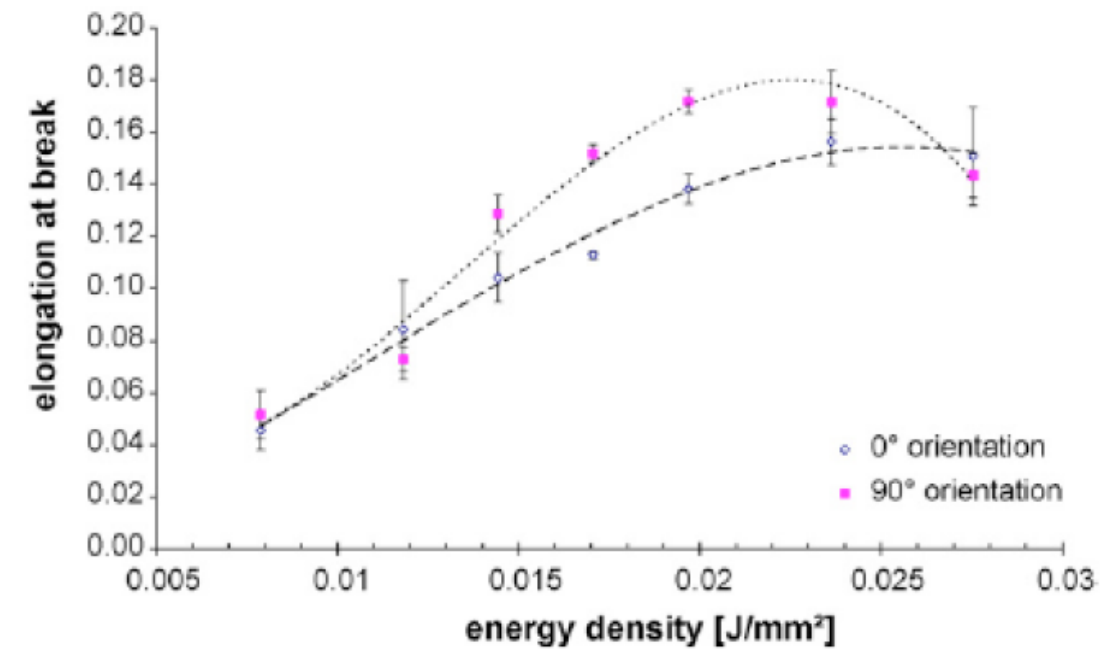

Figure 1 Effect of laser power on elongation at break in Nylon-12 (Caulfield 2006)

\section{Experimental Methodology}

Two thermal characterization techniques were used to investigate a laser sintering grade of nylon-12, PA2201 manufactured by EOS with average particle size of 56 $\mu \mathrm{m}$. The thermal property experiments attempted to understand the behaviour of the material from room 
temperature to the temperature range at which it fully degrades. In order to accomplish this DSC was first used to examine the thermal phase change properties up to $220^{\circ} \mathrm{C}$ and then TGA was performed to understand the effect of high temperature on the polymer chain stability.

DSC

Differential Scanning Calorimetry is a measurement method that records the heat flow during phase transitions. It is a common tool used in characterizing materials for laser sintering because it quantifies the melt and solidification temperatures associated with the crystalline phases. A TA Instruments Q200 was used to perform the thermal analysis on the nylon-12. The heating and sample preparation were completed according to the ASTM D3413-08 protocol. Powder mass samples were $6.6 \pm 0.1 \mathrm{mg}$ and two separate runs were completed on individual powder samples. The samples were put through a heat-cool-heat procedure from $20^{\circ} \mathrm{C}-220^{\circ} \mathrm{C}$ at $10^{\circ} \mathrm{C} / \mathrm{min}$. Samples were sealed inside an aluminium container and nitrogen gas was used as a coolant at a flow rate of $50 \mathrm{~cm}^{3} \mathrm{~min}^{-1}$. Results were analyzed using TA Universal Analysis software. An interpolated baseline was produced on each DSC plot noting maximum and minimum heat flow for both heating and cooling. The peak trough height measured from this line was used for creating the area of the section 
thus ascertaining the heat of fusion and crystallization (See Figure 2) Temperatures at the peaks were labelled as melt and crystallization points.

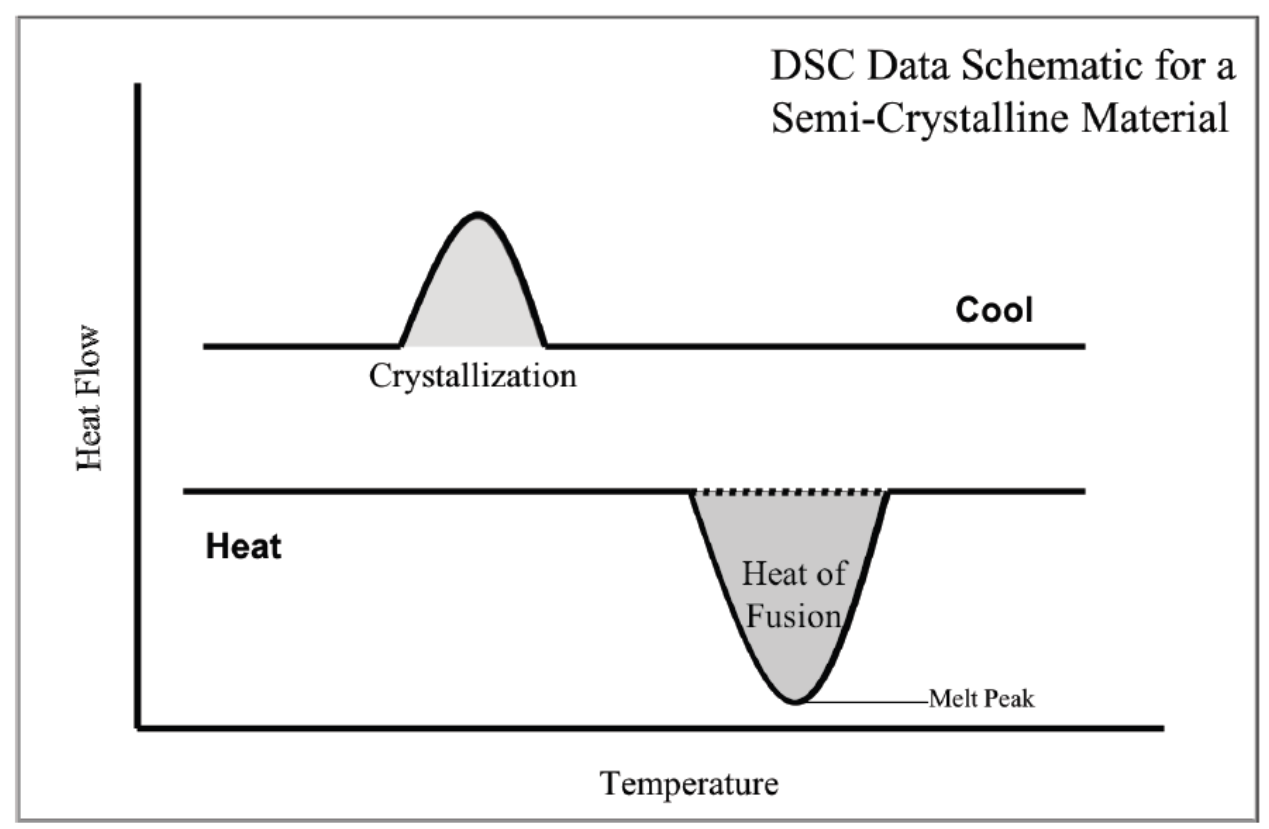

Figure 2 Typical DSC trace

TGA

Thermogravimetric analysis (TGA) is a common means of assessing degradation in materials. TGA is a measurement of weight gain/loss as a function of temperature and time using a highly sensitive microbalance (ASM 2003). Usually polymer samples ranging from 
10-20mg are examined from room temperature to decomposition or pyrolysis temperature in a nitrogen atmosphere (ASM 2003). This testing method allows for comparison not only between materials but also can give insight into lifetime predictions, moisture content, filler / inorganic content and volatility with a material.

Nylon-12 powder samples of approximately $10 \mathrm{mg}$ were heated from $30^{\circ} \mathrm{C}$ to $600^{\circ} \mathrm{C}$ in a 95\% nitrogen and 5\% oxygen atmosphere. This condition was chose because it offers a close model of the conditions seen inside a standard laser sintering machine. Three separate tests were performed on the material.

\section{Results}

\section{Polymer Characterization (DSC and TGA)}

Figure 3 shows the results from one run of the DSC for the material. Nylon-12 is a semicrystalline thermoplastic polymer and this is confirmed for this grade of material with the

existence of two distinct peaks for melting and solidification. Other more amorphous materials would not show sharp peaks and overlap between the areas. 


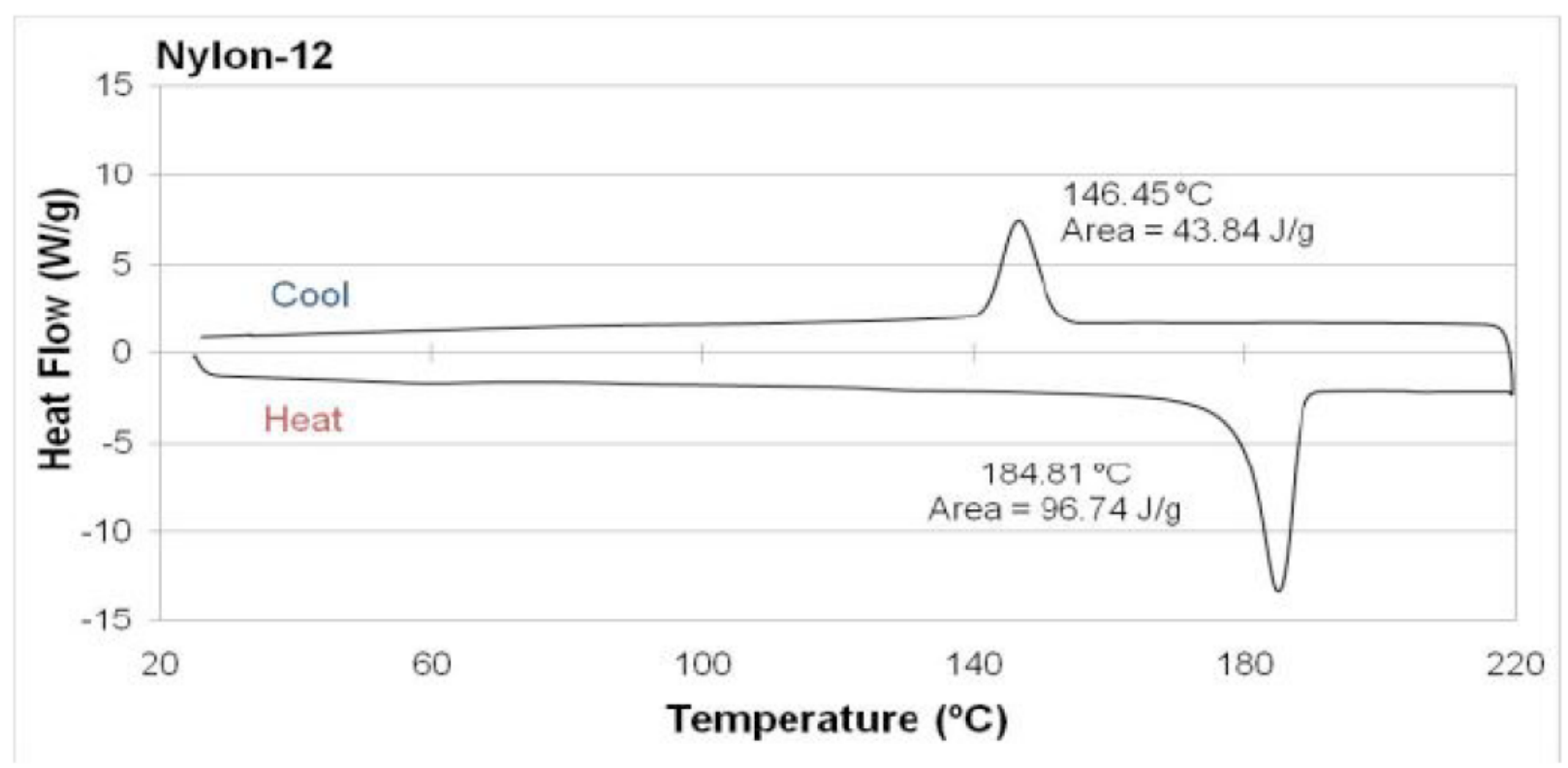

Figure 3 DSC thermograms for Nylon-12 powder

The melting point for this material is $184.8^{\circ} \mathrm{C}$ with a heat of fusion of $96.7 \mathrm{~J} / \mathrm{g}$. After the material has been melted it re-solidifies with a peak crystallization temperature of $146.5^{\circ} \mathrm{C}$. The solidification temperature is significantly lower than the crystalline melting temperature; this phenomenon is common in crystallisable polymers and is known as super cooling.

The results from the TGA experiments are shown in Figure 4. The heating cycle started from $30^{\circ} \mathrm{C}$ and Table 1 shows the temperatures with their corresponding weight loss 
percentages. The peak loss in mass occurs around $430^{\circ} \mathrm{C}$ and can be confirmed by the peak maximum in the derivative weight loss percentage curve (not shown here). However a deterioration of properties, due to conditions experienced in commercial processes, is likely to occur at much lower temperatures and for weight loss much less than $50 \%$. 


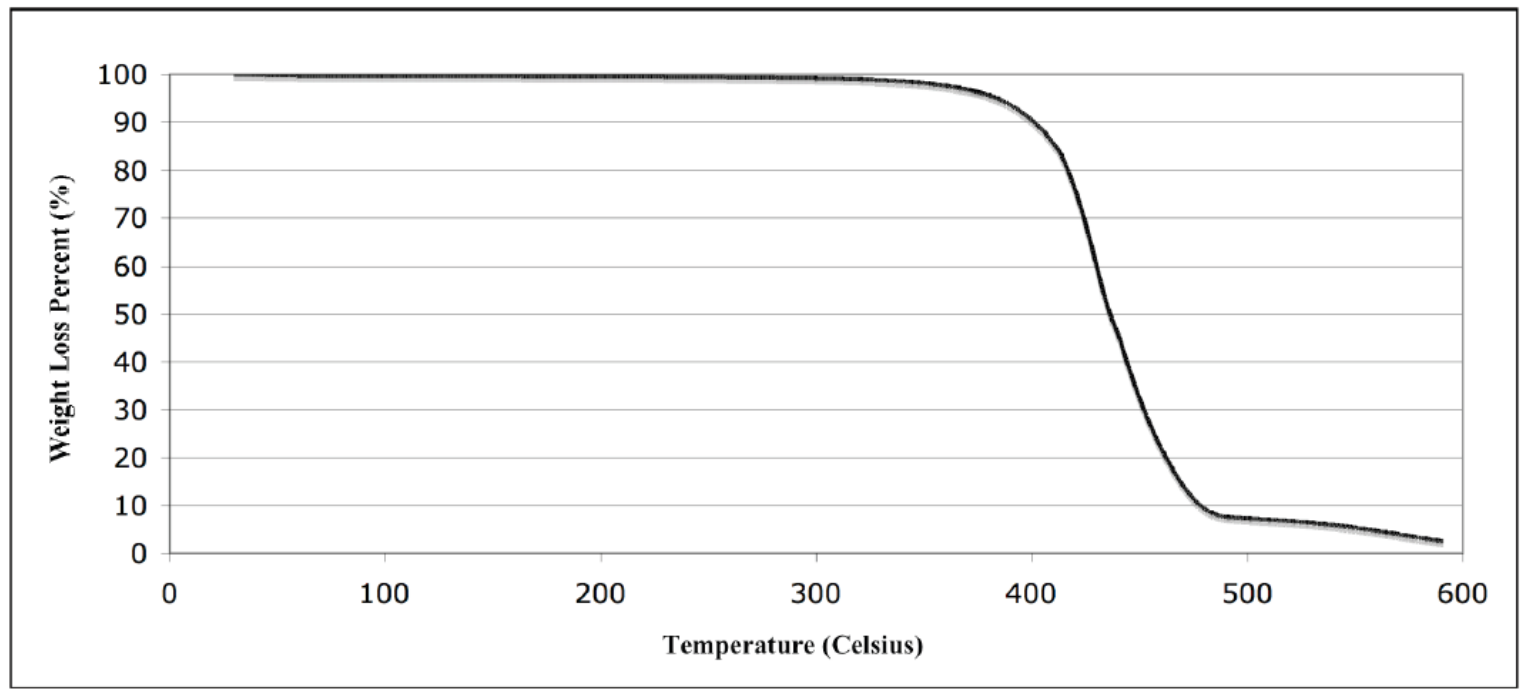

(a)

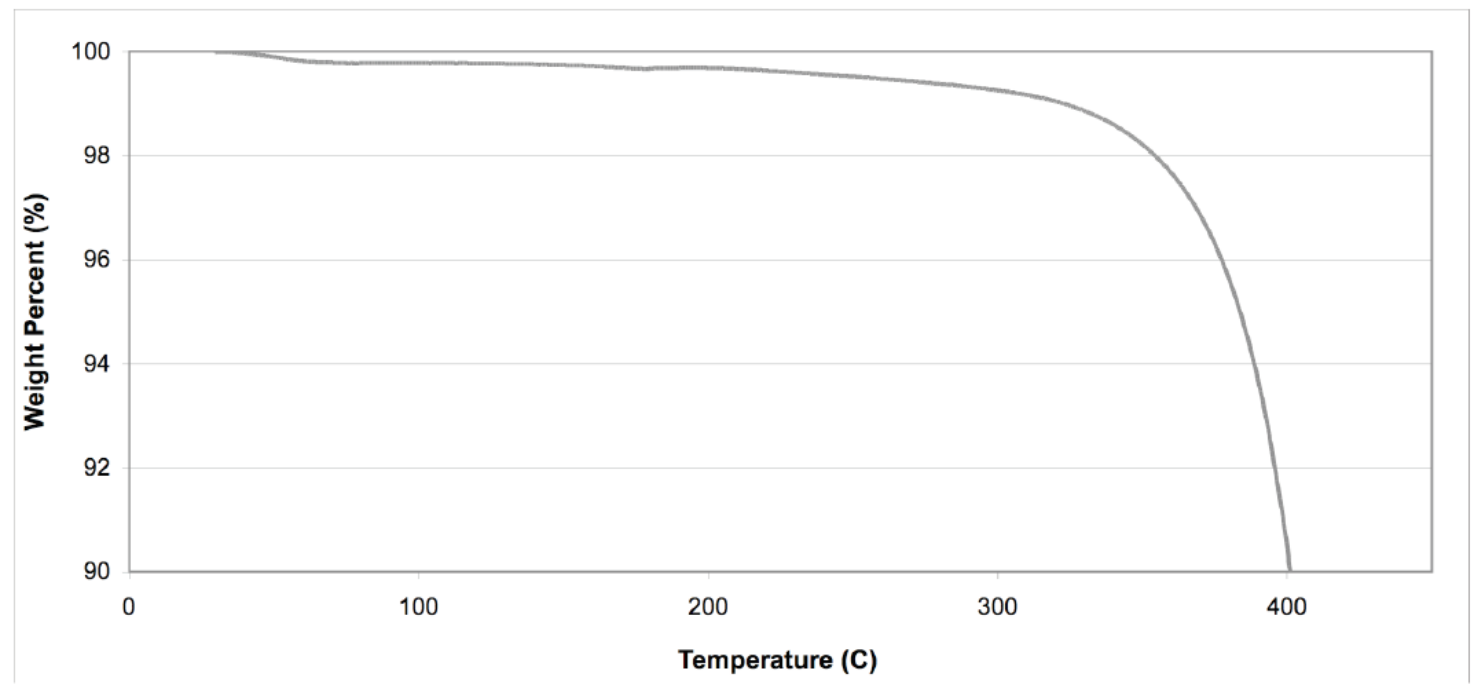

(b) 


\section{Figure 4 Nylon-12 TGA results: (a) Full Temperature Range; (b) Expanded to show $0-10 \%$ weight loss data}

Table 1 TGA Results for Nylon-12 - Summary

\begin{tabular}{|l|llll|}
\hline TGA Results & & & & \\
\hline Weight Loss Percent & $1 \%$ & $2 \%$ & $5 \%$ & $50 \%$ \\
\hline Temperature [C] & 322 & 353 & 383 & 436 \\
\hline
\end{tabular}

\section{Estimation of temperatures in laser sintering}

Given the small spot size and high speed of the laser inside the machine it is difficult to exactly identify an instantaneous material temperature. The approach taken in this work is an estimation method using the inputs from the Caulfield work to determine the temperature exposure of the Nylon-12 during sintering. This procedure is to verify that it is theoretically possible for the temperatures inside the machine to approach the regions of thermal stability for the polymer given the inputs used by Caulfield (2007). These values will then be compared with the experimental DSC and TGA work.

\section{Initial Constraints and Parameters}

The bed temperature used by Caulfield for the processing of the test specimens was $150^{\circ} \mathrm{C}$.

This is the initial temperature $T_{0}$. It is assumed that this is uniformly distributed over a 
layer of particles. This bed temperature is lower than normally used.; most nylon-12 processing utilizes a bed temperature from $170-178^{\circ} \mathrm{C}$. In many cases the temperature sensors tend to drift with use and actually bed temperatures will be different from what the operator input settings. For this reason a second set of calculations with the standard bed temperature of $175{ }^{\circ} \mathrm{C}$ will be shown as well. A conventional laser sintering nylon-12 material has a particle size of $\sim 56$ microns (EOS). For this estimation the single particle will act as the layer thickness because these particles will initially absorb the majority of the energy from the laser. Particles below will also melt but some laser power will be shielded by the particles above, thus creating a thermal gradient.

$$
\begin{array}{r}
\mathrm{T}(\mathrm{x}, \mathrm{y}, \mathrm{z}) \mid=\mathrm{T}_{\mathrm{o}} \\
\mathrm{T}_{\mathrm{o}}=150^{\circ} \mathrm{C}
\end{array}
$$

The energy density (Equation 1) data used for testing the mechanical properties are outlined in Table 2. These values are shown with their corresponding laser power. 
Table 2 Laser Sintering Parameters

\begin{tabular}{|l|l|l|l|l|l|l|l|l|}
\hline & Set 1 & Set 2 & Set 3 & Set 4 & Set 5 & Set 6 & Set 7 \\
\hline Laser Power $(\mathrm{W})$ & 6 & 9 & 11 & 13 & 15 & 18 & 21 \\
\hline Energy Density $\left(\mathrm{J} / \mathrm{mm}^{2}\right)$ & 0.00787 & 0.01181 & 0.01443 & 0.01706 & 0.01968 & 0.02362 & 0.02775 \\
\hline
\end{tabular}

The total amount of energy $\left(\mathrm{H}_{\text {laser }}\right)$ delivered to the material independent of the bed temperature is shown in equation 3 and will be expressed in units of $\mathrm{J} / \mathrm{g}$ (where $\delta$ is the density of the material and $\mathrm{z}$ is the layer thickness). It is unlikely that the material will absorb all of the incident energy delivered by the laser so an absorption fraction (A) is included. Therefore an absorption efficiency between zero and unity will be introduced. L is the energy density applied for a given laser power outlined in Table 1.

$$
H_{\text {laser }}=\frac{A L}{\delta z}
$$

The part bed temperature is held just below the melting point of the polymer. Therefore the energy applied by the laser supplies the additional energy required to initiate the melting phase, as temperature increases through the endothermic region (e.g. Figure 3). The 
enthalpy of melting $\Delta \mathrm{H}_{\text {fusion }}$ can be estimated from a DSC trace of the material and is equal to the area of the melting endotherm. Therefore the total energy that the laser provides to the material can be calculated using Equation 4 where $E_{\text {flow }}$ is the enthalpy required to raise unit mass of powder from bed temperature to sintering temperature

$$
E_{\text {flow }}=H_{\text {laser }}-\Delta H_{\text {fusion }}
$$

Beyond the melt temperature the thermal behaviour can be expressed as a function of the thermal diffusivity $(\alpha)$, density, thermal conductivity $(k)$ and the heat capacity $\left(C_{p}\right)$ in Equation 5.

$$
C_{p}=\frac{k}{\delta \alpha}
$$

A material's heat capacity is a temperature dependent variable however the region of interest for nylon- 12 is between $190-270^{\circ} \mathrm{C}$. In this range, an average heat capacity of $C_{p}^{\text {avg }}$ is used. The predicted increase in exposure temperature of the powder is therefore given in Equation 6. 


$$
\Delta T_{\text {laser }}=\frac{A E_{\text {flow }}}{C_{p}^{\text {avg }}}
$$

The maximum temperature, $\mathrm{T}_{\max }$, is calculated from the sum of the temperature induced by the laser and the bed temperature.

$$
T_{\max }=T_{b e d}+T_{\text {laser }}
$$

Using Equations 5 and 6 the maximum temperature experienced at various laser power settings was calculated and is shown in Table 4. The input values for these calculations are shown in Table 3. Table 4 shows that at a bed temperature of $150^{\circ} \mathrm{C}$ the lower energy laser passes would not provide enough energy to melt the polymer. The remaining calculations shown in Table 5 use the standard bed temperature of $175^{\circ} \mathrm{C}$ at various laser energy absorption rates. 
Table 3 Input Values for Laser Sintering Calculations

\begin{tabular}{|c|c|c|c|c|}
\hline \multicolumn{5}{|l|}{ Material Properties } \\
\hline & & nits & Condition & Conversion \\
\hline Melt Density & 0.88634 & $\mathrm{~g} \mathrm{~cm}^{-1}$ & Melt & 0.00088634 \\
\hline Specific Heat Capacity & 2500 & $\mathrm{~J} \mathrm{~kg}^{-1} \mathrm{C}^{-1}$ & $190-270 \mathrm{C}$ & 2.5 \\
\hline Particle Size & 56 & $\mu \mathrm{m}$ & & 0.056 \\
\hline Heat of Fusion & 96.74 & $\mathrm{Jg}^{-1}$ & & \\
\hline
\end{tabular}

Table 4 Estimation Results at $150^{\circ} \mathrm{C}$ bed temperature, for $100 \%$ absorption

\begin{tabular}{|lcccccccc|}
\hline Results at 100\% Absorptance at 150C & \multicolumn{10}{c|}{ Set } & Set 3 & Set 4 & Set 5 & Set 6 & Set 7 \\
\cline { 2 - 10 } & Set 1 & Set & 6 & 11 & 13 & 15 & 18 & 21 \\
\hline Laser Power (W) & 175 & 203 & 224 & 244 & 264 & 295 & 327 \\
Exposure Temperature (C) & & &
\end{tabular}

Table 5 Estimation Results with varying absorption factors $\left(175^{\circ} \mathrm{C}\right.$ bed temperature)

\begin{tabular}{|c|c|c|c|c|c|c|c|}
\hline \multirow{2}{*}{\multicolumn{8}{|c|}{ Results at $100 \%$ Absorptance at $175 \mathrm{C}$}} \\
\hline & Set 1 & Set 2 & Set 3 & Set 4 & Set 5 & \multicolumn{2}{|l|}{ Set 6} \\
\hline Laser Power (W) & 6 & 9 & 11 & 13 & 15 & 18 & 21 \\
\hline Exposure Temperature (C) & 200 & 228 & 249 & 269 & 289 & 320 & 352 \\
\hline \multicolumn{8}{|c|}{ Results at $80 \%$ Absorptance at 175} \\
\hline & Set 1 & Set 2 & Set 3 & Set 4 & Set 5 & Set 6 & Set 7 \\
\hline Laser Power (W) & 6 & 9 & 11 & 13 & 15 & 18 & 21 \\
\hline Exposure Temperature (C) & 196 & 220 & 238 & 255 & 272 & 298 & 326 \\
\hline \multicolumn{8}{|c|}{ Results at $60 \%$ Absorptance at 175} \\
\hline & Set 1 & Set 2 & Set 3 & Set 4 & Set 5 & Set 6 & Set 7 \\
\hline Laser Power (W) & 6 & 9 & 11 & 13 & 15 & 18 & 21 \\
\hline Exposure Temperature (C) & 190 & 207 & 219 & 231 & 244 & 262 & 281 \\
\hline
\end{tabular}


A lower bound value of $60 \%$ absorption was chosen in this case because selecting a value below this would not result in sufficient energy transfer to melt the particles, in a single pass of the laser at low wattages and at $175^{\circ} \mathrm{C}$. It is suspected that even with multiple passes there could be difficult sintering due to the high melt viscosity at temperatures just above the melting point.

Figure 5 shows the graphical representation of the results predicted from each of the laser power values and absorption rates. There are limitations to this methodology including the fact that the laser interaction time with the powder is only fractions of a second, so the exposure cycle is small. Secondly the shape of the particle can lead to high exposure on the top surface for the material and thermal conductivity within the particle will delay the transfer of heat throughout the particles and deeper into successive layers. Use of process heat transfer theory (beyond the scope of this paper) would be required to estimate the ratio of external to internal heat flow. An additional variable that can be incorporated will be the scan count or number of times the laser hits the same area of powder. For this paper the estimation of scan count will be held at 1 . 


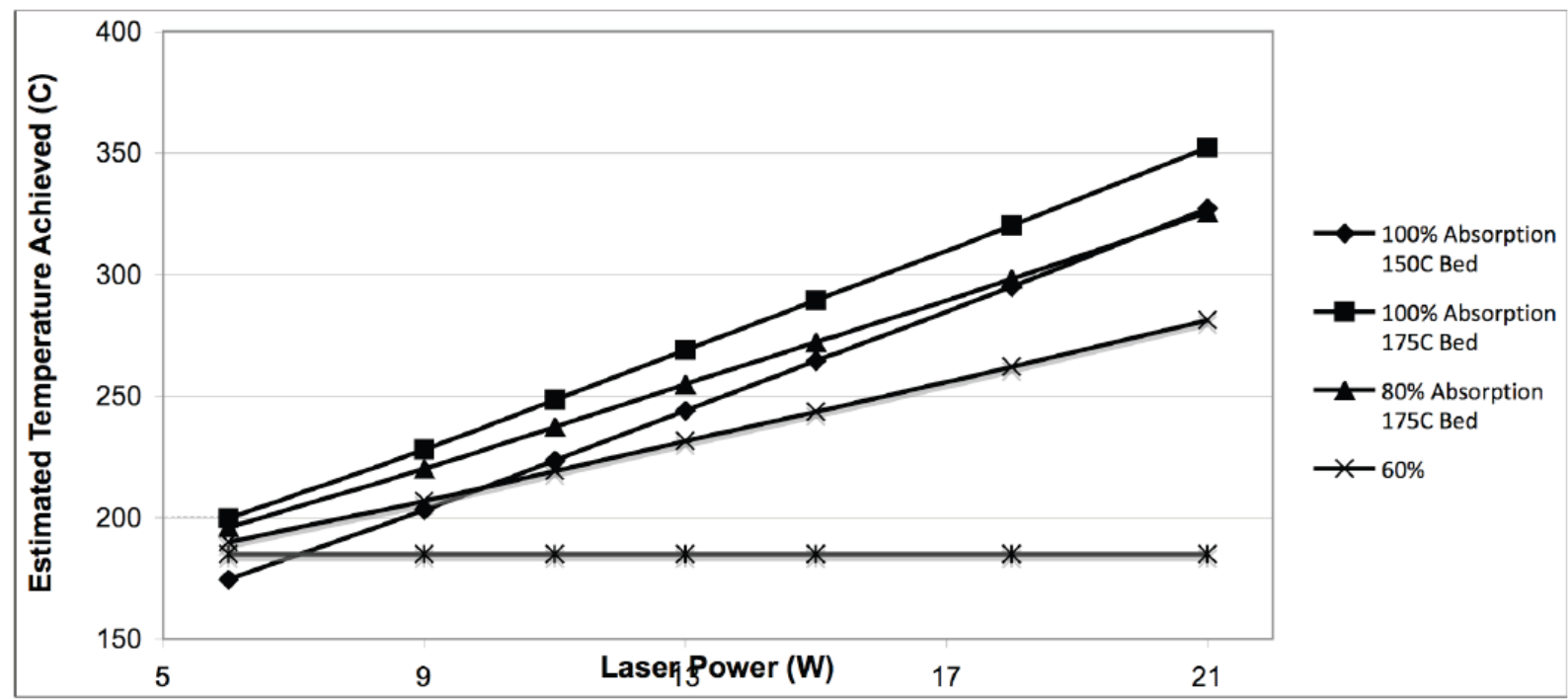

Figure 5 Estimation of powder temperature at various laser powers and absorption rates

\section{Discussion}

In order to achieve high quality laser sintering parts it is necessary to fully melt the polymer material to initiate liquid phase flow necking and consolidation. As the Caulfield (2007) data suggests, the more fully heated nylon-12 the stronger the predicted properties, since viscosity decreases at higher melt-state temperatures. However if the temperature developed on the particles is too high, an opposing effect can occur due to degradation, resulting in loss of properties. Using the data from the TGA and temperature calculations discussed in the first part of this paper the term 'stable sintering region' is proposed. This 
term is outlined graphically in Figure 6 and is the result of combining the results from the DSC and TGA tests. The DSC is used to track the endothermic melting temperatures of the nylon (minimum sintering requirement) and the TGA analysis gives an upper temperature limit for the material. The upper limit for process temperature is material-specific and needs to be carefully specified from knowledge of chemical degradation mechanism and the sensitivity of reaction kinetics to factors such as temperature and exposure time. TGA can be used as part of the process whereby the upper temperature limits can be proposed.

Using the Caulfield data it was shown that temperatures inside the laser sintering machine can reach levels where material mass loss is experienced. The temperatures induced at higher laser wattages used by Caulfield were in the regions where mass loss was seen with the TGA even at absorption rates as low as $80 \%$, where surface temperatures may reach over $315^{\circ} \mathrm{C}$. The information from these material characterization tests can then be directly used with the inputs from the laser sintering machine to identify the laser power window that is available, given the sintering conditions and bed temperature. 


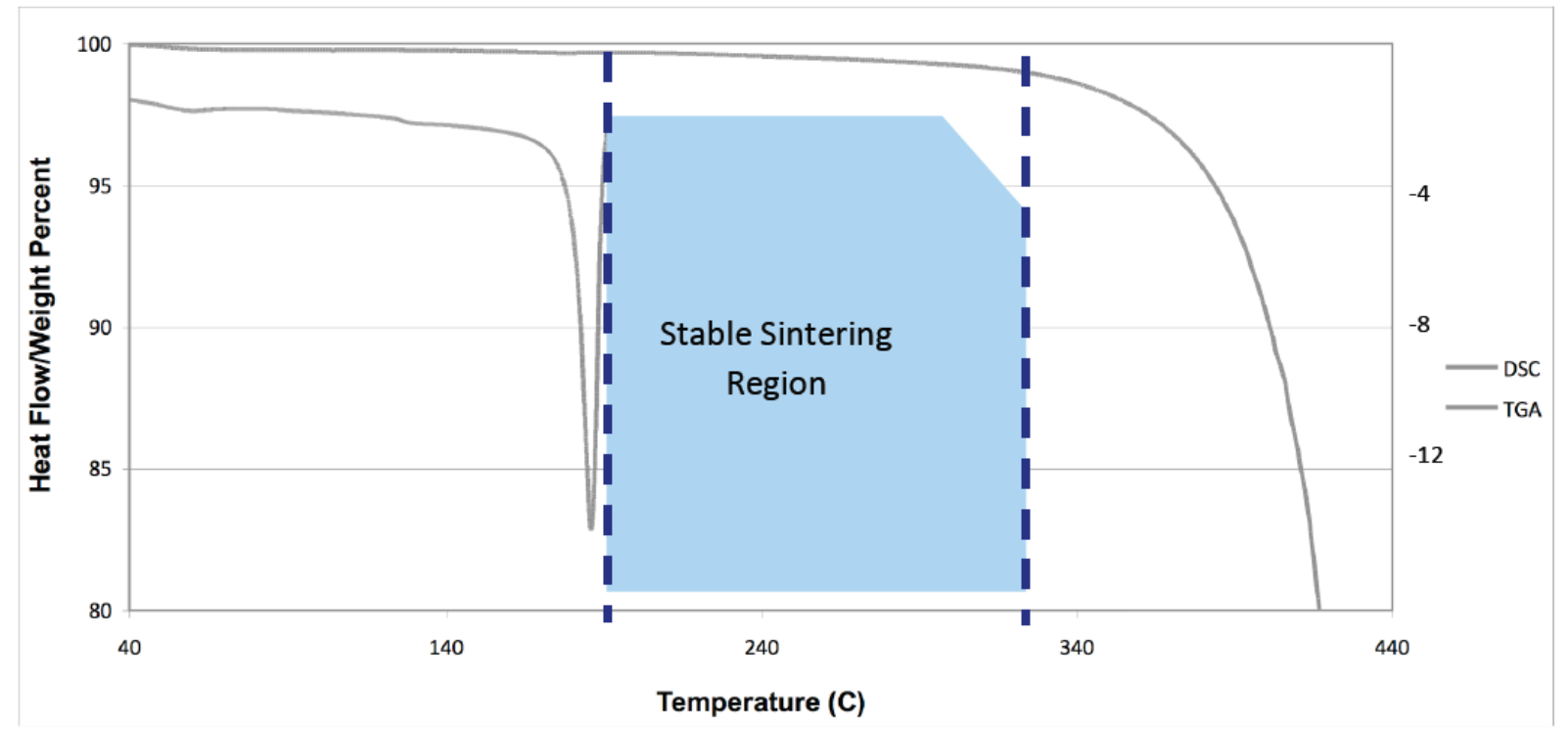

Figure 6 'Stable sintering region' proposed for semi-crystalline polymers

Figure 7 is a visual representation of the thermal profile of nylon-12 powder from room temperature to the upper degradation limit. The diagram is a laser sintering specific explanation of the process window divided into four sections. First the material is heated to a point at which it starts to melt and flow, once above the melting endotherm. The stable sintering region is the temperature range in which the material can be processed without being degraded, with viscosity as a key physical property within this region. Once the material is sintered by the laser the temperature drops and the material consolidates in 
cooling mode. The material stays in the melt phase below the melt temperature until crystallization. This defines the supercooling window. Typical laser sintering machines set the bed temperature in this zone to encourage more fully sintered parts and to avoid part distortion or curling, in the part bed.

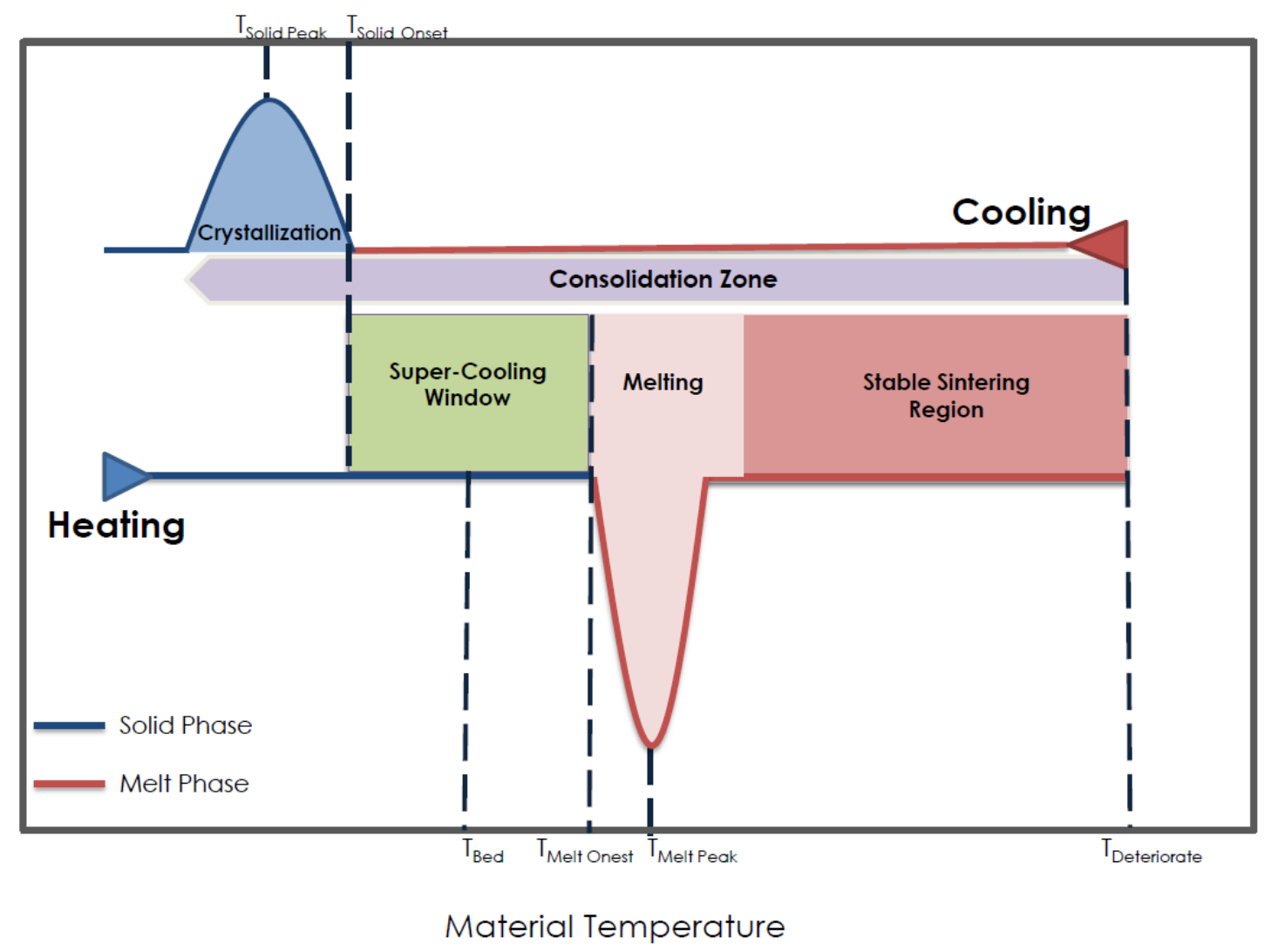

Figure 7 Laser sintering window, for semi-crystalline polymers 


\section{Conclusions}

Understanding the parameter design and optimization of the laser sintering process is valuable for both end users and material manufacturers. The ability to process at varying conditions such as laser power, bed temperature, or scan count is one of the strengths of the machine and allows the user to tune the mechanical properties of their parts. However the introduction of a 'stable sintering region' is a tool that can be used to further explore different materials. Previously the main thermal characterization tool used for laser sintering materials was DSC however with the addition of a TGA test more information on the polymer can be used to identify behaviour throughout the full range of temperatures seen in the process, in both heating and cooling mode.

The terms 'Stable Sintering Region' and 'Laser Sintering Window' are platform concepts and more work is intended to identify the exact TGA methodology that gives a complete mass deterioration picture, for the nylon-12 polymers currently used. Other factors to be investigated include varying atmospheric conditions, heating speed, and isothermal behaviour. However the initial work done in a standard protocol does deliver valuable information for the user. The biggest limitation of the work is the approximate nature of temperature calculations achieved during laser sintering. The method reported here 
develops from previous work done by other researchers as a basis for estimating the conditions seen in the machine. Nevertheless, estimations of temperature achieved support the work done by earlier authors on the deterioration of mechanical properties with higher laser powers.

The concepts illustrated in Figures 6 and 7 can be applied as an informative picture of the laser sintering process for nylon-12 but are certainly not limited to one material. The combination of TGA and DSC testing can be a valuable tool for identifying new materials that could be used in the process. The key parameters such as melt temperature, solidification temperature, supercooling zone, and stable sintering zone can be easily diagrammed and analyzed as a material selection tool.

This work was supported by the Loughborough University Innovative Manufacturing and Construction Research Centre Project 251. 


\section{Sources}

ASM International. Characterization and Failure Analysis of Plastics. Ohio: ASM International, 2003, p359-382.

Caulfield, B., McHugh PE, Lohfeld S. Dependence of Mechanical Properties of polyamide components on build parameters in the SLS process; Journal of Materials Processing Technology 2007. 182:477-488 (accessed 25 January 2011).

Colin, X and Verdu J., Polymer degradation during processing. CR Chimie 2006. 13801395.

EOS Data Sheet PA 2201 http://www.eos.info/en/home.html (Accessed 2010).

Ghosh, S., Rastgir D., Bhowmick A., Mukinda P. Thermal degradation and aging of segmented polyamides; Polymer Degradation and Stability 2000; 67:427-436.

Herrera, M. Matushchuk G., KettrupA. Main products and kinetics of the thermal degradation of polyamides. Chemosphere 2001; 42:601-607. 
Hopkinson, N., Hague, R.J.M. and Dickens, P.M. (eds), Rapid Manufacturing and Industrial Revolution for the Digital Age, Chichester: John Wiley and Sons Ltd, 2005, p285, ISBN 0-470-01613-8.

Hopkinson, N., Majewski, C.E. and Zarringhalam, H, "Quantifying the degree of particle melt in Selective Laser Sintering®", CIRP Annals - Manufacturing Technology, 58(1), 29th March 2009, pp 197-200

Williams, JD. Deckard, C. Advances in modelling the effects of selected parameters on the SLS process; Rapid Prototyping Journal 1998; 4:2:90-100.

Zarringhalam, H., Majewski, C.E. and Hopkinson, N., Degree of Particle Melt in Nylon-12 Selective Laser Sintered Parts, Rapid Prototyping Journal 2009; 15:2:126-132. 